\title{
Primary observations of solar filaments using the multi-channel imaging system of the New Vacuum Solar Telescope
}

\author{
Zhi Xu, Zhen Y. Jin, Fang Y. Xu and Zhong Liu \\ and the NVST team \\ Fuxian Solar Observatory, Yunnan Observatory, \\ Chinese Academy of Science, Kunming, 650011 \\ email: xuzhi@ynao.ac.cn
}

\begin{abstract}
The New Vacuum Solar Telescope (NVST) is a new generation ground-based solar facility of China. One of the post-focus instruments is the multi-channel high-resolution imaging system, which is designed to simultaneously observe the dynamic gas motion in the solar photosphere and chromosphere. Since October of 2010 it has been operational in the NVST and some necessary updates were performed in past 2 years. Here we first give a general introduction of this system, and then we exhibit one near-limb observation of solar filaments obtained using this system. By this communication, we would like to show the potential ability to perform the high resolution observation of solar filaments (prominences) using the multi-channel imaging system in the NVST.
\end{abstract}

Keywords. ground-based solar facilities, the NVST, solar prominences

\section{Introduction}

The New Vacuum Solar Telescope (NVST) belongs to a new generation of large and high-technology solar facilities of China. It is designed to observe the Sun in the range of $0.3-2.5$ micron using high resolution imaging systems and spectrometers with a polarization analyzer. The NVST is a 1-m diameter telescope (a pure aperture of 980 millimeter) on an alt-azimuthal mount with an effective focal length of 45 meter (Liu and $\mathrm{Xu}, 2011$ ). The NVST is located at the northeast bank of Fuxian Lake of Yunnan province. It is the most important facility of the Fuxian Solar Observatory (FSO). See the website $h t t p: / / f$ so.ynao.ac.cn for more information about the FSO.

The first-light of the NVST came in October 2010. Two instruments are operational. One is the multi-channel high-resolution imaging system, the other one is the multiwavelength spectrometer in visible lines (Wang et al., 2013). These two systems are designed and arranged to be perpendicular to each other. Fig. 1 shows the present layout of the instruments in the focus plane. In this way, the imaging system can simultaneously serve as the slit-jaw recorder of the spectrometers.

The imaging system comprises 5 channels, the work wavelengthes and the main properties of each channel are summarized as follows:

- $\mathrm{H} \alpha$ line, to monitor magnetic structures in the chromosphere. The central wavelength can be tunable in the range of $6562.8 \pm 4 \AA$ and the full bandpass width is $0.25 \AA$ (e.g., giving a FWHM of an order of $11 \mathrm{~km} / \mathrm{s}$ ). The time cadence of Level-1 data (post-processed based on a luck image selection algorithm ) is about 12 seconds.

- $\mathrm{G}$ band, to indicate small magnetic structures in the deep photosphere (Sütterlin et al., 2001). The central wavelength is at $4300 \AA$ and the full bandpass width is $10 \AA$. 


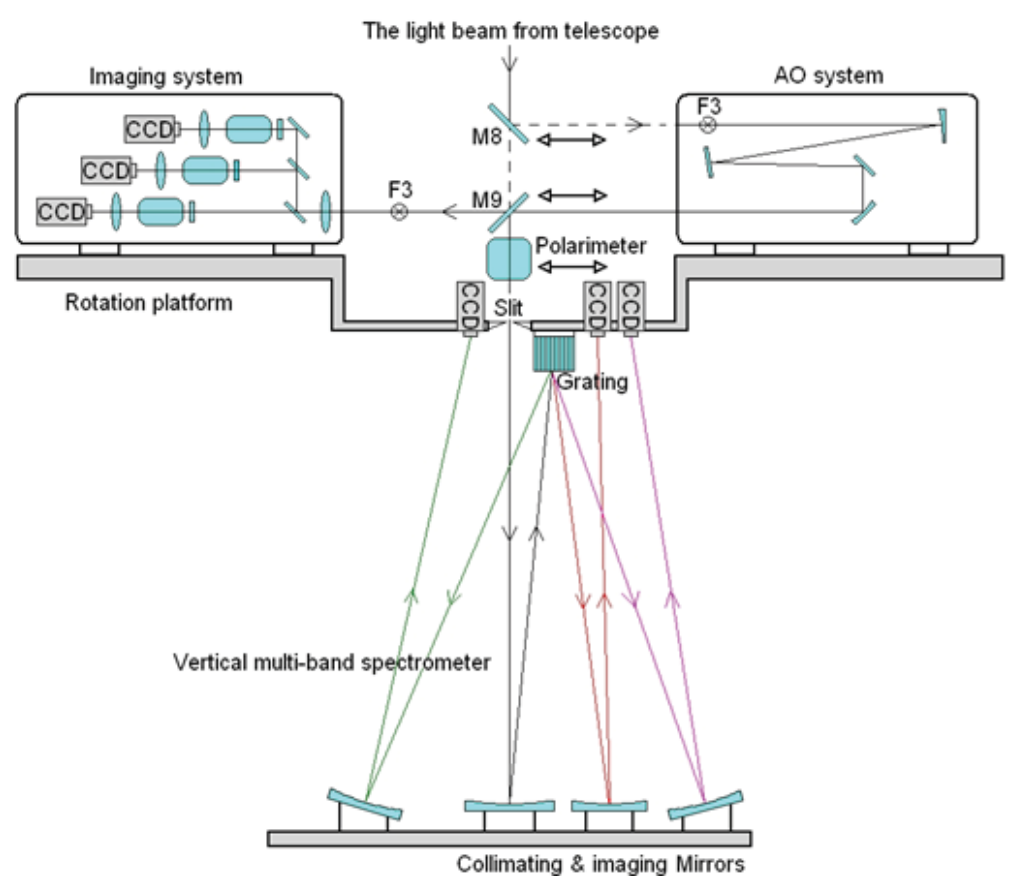

Figure 1. Layout of the instruments in the focal plane of the NVST. The main instruments include a multi-channel high-resolution imaging system, a vertical multi-wavelength spectrometer and a low-level AO system.

- $\mathrm{TiO}$ band, sensitive to the temperature, to allow easy detect bright points in granular lanes or umbral dots. It is centered at $7058 \AA$ and the full bandpass width is $10 \AA$. The time cadence of Level-2 data (post-processed based on the speckle reconstruction) is about 40 seconds.

- Ca II $8542 \AA$, to monitor magnetic structures in the upper chromosphere. The central wavelength can be tunable in the range of $8542 \pm 10 \AA$ and the full bandpass width is $0.2 \AA$ (an alternative width is $0.4 \AA$ ).

- He I $10830 \AA$, a near infrared proxy of coronal holes, to explore magnetic fields in the low corona. The parameters need to be determined.

Although up to now only three channels including the $\mathrm{H} \alpha$, $\mathrm{TiO}$ and G-band are being used, the observations impressively demonstrate the high-resolution capability of the NVST (as shown in Fig.2). In particular, the post-processing with the speckle masking method produces the results with the resolution close to the diffraction limit. We use the speckle masking method of Weigelt (1977). It is adapted by Liu et al. (1998). To do so, we have to take a large number of short-exposure images $\left(t_{H \alpha} \leqslant 20 \mathrm{~ms}, t_{\text {Tio }} \sim 1 \mathrm{~ms}\right)$. Using the large number of frames (200 frames) and a statistical algorithm, we are able to reconstruct one frame with the whole field of view ( $\approx 3$ arcmin). There are normally three processive levels of the data reduction. Level-0 represents the raw status. Level-1 is achieved based on a luck image selection algorithm, followed by the dark current and flat field modification. Level-2 is reconstructed by a speckle masking method. Quick look of the daily observation in the $\mathrm{H} \alpha$ channel (Level-1) can be viewed from the website of http : //fso.ynao.ac.cn/dataarchive_ql.aspx. 

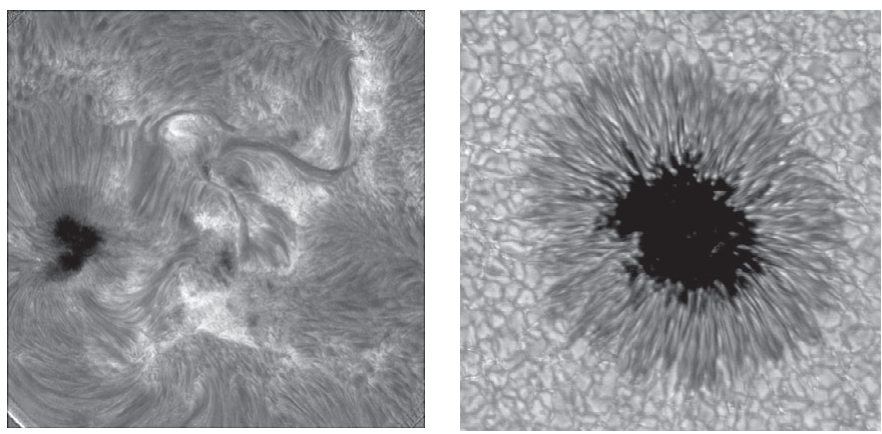

Figure 2. Observations (Level-1 data) in the $\mathrm{H} \alpha$ line center (left) and $\mathrm{TiO}$ band (right) channels. The spatial resolution are about 0.165 and 0.04 arcsec/pixel, respectively.
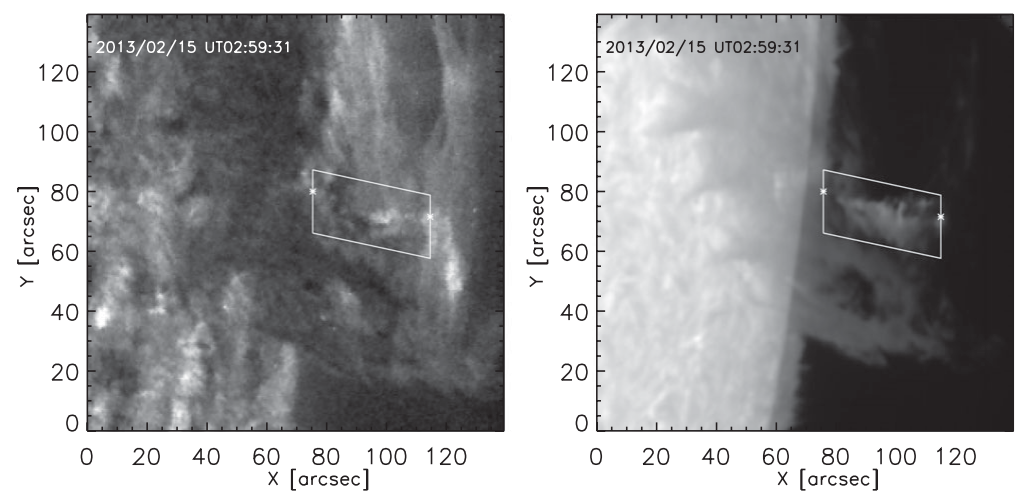

Figure 3. Simultaneous observations of a near-limb filament taken by the AIA/SDO at the 304 $\AA$ channel (left) and the NVST at the $\mathrm{H} \alpha$ channel (right) on Feb. 15 2013. The spatial resolution of the NVST is reduced to be the same as the AIA image. A parallelogram delimitates a dynamic structure where successive outflows are observed.

\section{Filaments observations in the $\mathbf{H} \alpha$ channel}

Using this imaging system, we carried out several time observations about solar filaments or prominences in both the $\mathrm{H} \alpha$ and $\mathrm{TiO}$ channels. The $\mathrm{H} \alpha$ Lyot filter was updated in 2013 April. The transmission rate is quite increased but the main properties, such as the tunable central wavelength and the bandpass width are consistent. As we know, in order to extract some physical information, e.g., the Doppler velocity, from such narrowband $\mathrm{H} \alpha$ filtergrams, one needs to obtain a quasi-profile by scanning through this line. Considering the requirement of the data post-processing, we needs to take at least 200 frames at one wavelength scan position. Therefore,we normally use 3 samplings across the profile to obtain the cadence below 1 minute. The default sampled wavelengths are at $-0.7 \AA, 0 \AA$ and $+0.7 \AA$ off-band positions, which can be certainly changed by observers.

In this letter, we would like to present one high resolution observation of a near-limb filament obtained on Feb. 15 2013. It is observed in the $\mathrm{H} \alpha$ line center without wavelength scan to achieve a temporal resolution of 12 seconds. We took observations for more than one and a half hours in the NVST. In Fig. 3, we exhibit one $\mathrm{H} \alpha$ frame and compare with the simultaneous observation taken by the AIA/SDO (Lemen et al., 2012) in the $304 \AA$ channel (where emission is predominantly from He II). The $304 \AA$ channel is used to monitor the chromosphere and transition region (see the review of Labrosse et al., 2010). The resolution in the $\mathrm{H} \alpha$ is about 0.165 arcsec/pixel, which is 3.6 times as high as that of the AIA $304 \AA$ image. The main body and the barbs/legs of the filament are stable. By 


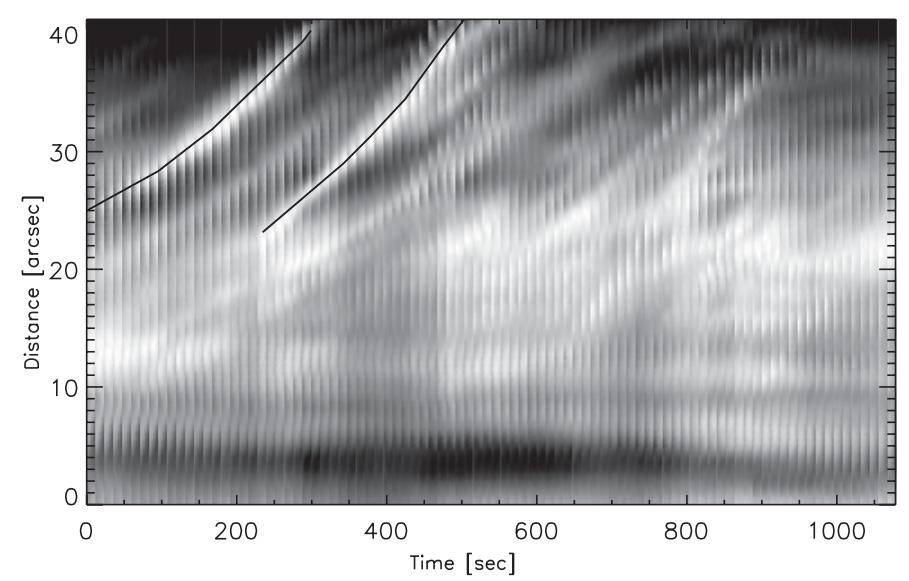

Figure 4. Time-space plot of a slice inside the parallelogram shown in Fig. 3. At each time, we take a slice of 1 arcsec wide and 40 arcsec long. Two black curves indicate polynomial fit results.

contrast, an adjacent structure delimitated by a parallelogram is very dynamic. It is seen that in the $\mathrm{H} \alpha$ line center, some fragments are going outwards along the local vertical direction for more than a half hour. We investigate the proper motion of these fragments by showing the space-time diagram of a slice of 1 arcsec wide and 40 arcsec long inside this parallelogram. The results is plotted in Fig. 4. We deduce that the proper motion can reach up to $50-70 \mathrm{~km} / \mathrm{s}$. In the AIA $304 \AA$ movie, it is difficult to recognize this motion due to a low resolution. Additional observations in the $193 \AA$ channel imply that this structure exhibits a rotating feature. Combining the NVST and SDO observations, we find that this outflow motion is approximately along a spiral axis of this rotating feature. More detailed analysis is beyond the content of this paper.

In conclusion, by the communication, we would like to demonstrate the potential capability of the multi-channel imaging system installed in the NVST to observe filaments or prominences with a high resolution. In addition, the imaging system of the Ca II 8542 $\AA$ line is now on trail. The remaining He I $10830 \AA$ channel will successively follow in 2014. We expect fruitful observations in future.

\section{Acknowledgement}

We thank the NVST instrument team for their technique support. SDO is a project of NASA. Xu Z. gratefully acknowledges the anonymous referee and the support of K.C.Wong Education Foundation, Hong Kong and the National Natural Science Foundations of China (grants No. 11103075).

\section{References}

Labrosse, N., Heinzel, P., Vial, J.-C. et al. 2010, Space Science Review, 151, 243

Lemen, J. R., Title, A., Akin, D. J. et al. 2012, Sol. Phys., 275, 17

Liu, Z., Qiu, Y., \& Lu, R. 1998, SPIE, 3561, 326

Liu, Z. \& Xu, J. 2011, in First Asia-Pacific Solar Physics Meeting, ASI Conference Series, edited by Arnab Rai Choudhuri \& Dipankar Banerjee, 2, 9

Weigelt, G. P. 1977, Optics Communications, 21, 55

Wang, R., Xu, Z., Jin, Z. Y. et al. 2013, Research in Astronomy and Astrophysics, 13, 1240

Sütterlin, P., Hammerschlag, R. H., Bettonvil, F. C. M. \& Rutten, R. J. 2001, in 20th International Sacramento Peak Summer Workshop, ASP Condference Series, edited by Sigawrth, M., 236, 431 